\title{
Homology modeling and virtual screening approaches to identify potent inhibitors of VEB-1 $\beta$-lactamase
}

\author{
Abdelmonaem Messaoudi, Hatem Belguith and Jeannette Ben Hamida
}

\author{
* Correspondence: \\ messaoudiabdelmonemster@gmail. \\ com \\ Unité de Protéomie Fonctionnelle \\ and Biopréservation Alimentaire, \\ Institut Supérieur des Sciences \\ Biologiques Appliquées de Tunis, \\ Université Tunis El Manar, 09, Rue \\ Docteur Zouheïr Safi - 1006, Tunis, \\ Tunisia
}

\begin{abstract}
Background: bla $a_{V E B-1}$ is an integron-located extended-spectrum $\beta$-lactamase gene initially detected in Escherichia coli and Pseudomonas aeruginosa strains from southeast Asia. Several recent studies have reported that VEB-1-positive strains are highly resistant to ceftazidime, cefotaxime and aztreonam antibiotics. One strategy to overcome resistance involves administering antibiotics together with $\beta$-lactamase inhibitors during the treatment of infectious diseases. During this study, four VEB-1 $\beta$-lactamase inhibitors were identified using computer-aided drug design.

Methods: The SWISS-MODEL tool was utilized to generate three dimensional structures of VEB-1 $\beta$-lactamase, and the 3D model VEB-1 was verified using PROCHECK, ERRAT and VERIFY 3D programs. Virtual screening was performed by docking inhibitors obtained from the ZINC Database to the active site of the VEB-1 protein using AutoDock Vina software.

Results and conclusion: Homology modeling studies were performed to obtain a three-dimensional structure of VEB-1 $\beta$-lactamase. The generated model was validated, and virtual screening of a large chemical ligand library with docking simulations was performed using AutoDock software with the ZINC database. On the basis of the dock-score, four molecules were subjected to ADME/TOX analysis, with ZINC4085364 emerging as the most potent inhibitor of the VEB-1 $\beta$-lactamase.
\end{abstract}

Keywords: VEB-1 $\beta$-lactamase, Homology modeling, Virtual screening, Docking, Inhibitor

\section{Background}

bla $a_{\mathrm{VEB}-1}$ was identified in 1996 from an Escherichia coli strain isolated from a Vietnamese patient. Subsequent analysis demonstrated that $b a_{\mathrm{VEB}-1}$ is both plasmid- and integronlocated [1,2]. Among the different Ambler class A expanded-spectrum $\beta$-lactamase (ESBL) genes, bla $a_{\mathrm{VEB}-1}$ is considered to be "emerging"; it has been detected in several Gram-negative organisms including Enterobacteriaceae and Pseudomonas aeruginosa $[3,4]$, and in multiple countries including France, Spain, Algeria, Turkey, Canada, Korea, Thailand and Tunisia [5-10]. Furthermore, P. aeruginosa isolates producing the VEB-1a variant, which differs from VEB-1 by only a single amino acid located in the leader peptide of the pre-mature protein, have been identified in Kuwait and India [11,12]. VEB-1 has high amino-acid identity to PER-1 and PER-2 (38\%) EBSLs, and confers high-level 
resistance to ceftazidime, cefotaxime and aztreonam [13]. The bla $a_{\mathrm{VEB}-1}$ gene was characterized in an unusual genetic environment in $P$. aeruginosa isolates from India and Bangladesh, and in P. stuartii from Algeria. Rather than having a typical class 1 integron structure, in these isolates bla VEB-1 $_{1}$ is flanked by identical 135-bp sequences, termed repeated elements (Res), which are bracketed by two truncated 3 '-conserved class 1 integron sequences in direct repeat [14].

There are currently no clinically useful inhibitors of VEB-1 $\beta$-lactamase. However, several studies have been undertaken using a variety of experimental inhibitors against other Ambler class A ESBLs. Clavulanic acid, the first $\beta$-lactamase inhibitor introduced into clinical medicine, was isolated from Streptomyces clavuligerus during the 1970s [15]. Clavulanate (the salt form of the acid in solution) presented with little antimicrobial activity in isolation, but when combined with amoxicillin, it significantly lowered amoxicillin MICs against Staphylococcus aureus, Klebsiella pneumoniae, Proteus mirabilis and E. coli [16]. Sulbactam and tazobactam are penicillinate sulfones developed as synthetic compounds in 1978 and 1980, respectively $[17,18]$. Class A $\beta$-lactamase is inhibited to comparable

levels by moxalactam, imipenem and cefoxitin.

The crystal structure of VEB-1 $\beta$-lactamase has not been described. Determining the three-dimensional (3D) structure of this molecule would assist in the discovery of more potent inhibitors, particularly in the application of structure-based virtual screening to identify lead compounds. To this end, a homology model of the 3D structure of VEB-1 protein was produced and a computational docking process was used to identify a series of potent inhibitors from the ZINC Database to allow VEB-1 to be compared with other Class A $\beta$-lactamase complexes.

\section{Methods}

Template identification and protein homology modeling

Searching the RCSB Protein Data Bank (http://www.rcsb.org/) confirmed that the tertiary structure of VEB-1 $\beta$-lactamase was not publicly available. The complete $E$. coli VEB-1 $\beta$-lactamase protein sequence, which consists of 299 amino acids and has a calculated molecular weight of $33.7 \mathrm{kDa}$, was retrieved from the UniProtKB database (http://www.uniprot.org/) (accession number Q7BVU7). BLASTP [19] was used to identify homologs in the RCSB Protein Databank [20]. Accordingly, the crystal structure of PER-1 $\beta$-lactamase from $P$. aeruginosa (PDB ID: 1E25), which has $40 \%$ sequence identity to VEB-1, was selected as the template [21]. To analyze sequence conservation, the VEB-1, PER-1, CTX-M and Toho-1 sequences were aligned. Gaps were inserted into the sequences to discover an optimal alignment, as presented in Figure 1A. The 3D structure of VEB-1 was modeled using the SWISS-MODEL tool [22] in the ExPASy Bioinformatics resource portal [23], and viewed using Swiss PDB Viewer v 4.0.1 software [24].

\section{Model optimization and evaluation}

Protein models generated using homology modeling frequently produce unfavorable bond lengths, bond angles, torsion angles and contacts. Therefore, it was essential to 

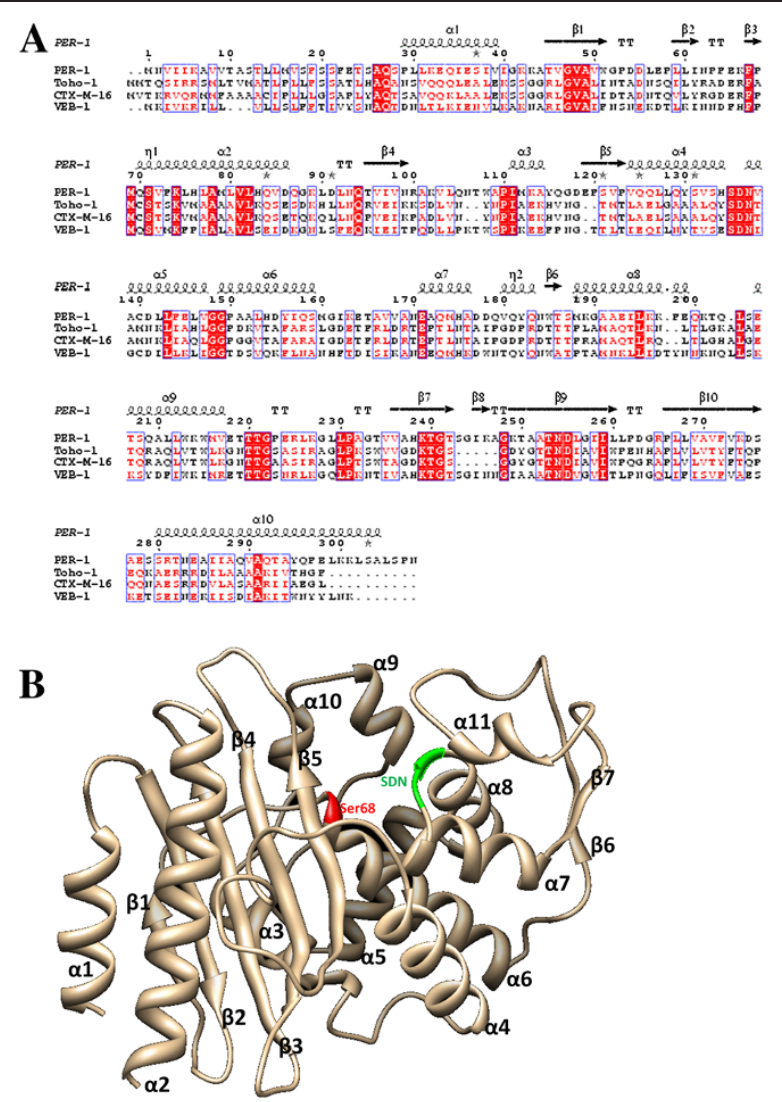

Figure 1 Overall structure of VEB-1 and its sequence alignment with its homologue proteins. A. Sequence alignment of VEB-1 with PER-1, Toho-1 and CTX-M-16. The second structure assignment of PER-1 is labeled on the top of the sequences. B. Cartoon representation of the overall structure of VEB-1 is in light orange color. The serine active-site is colored in red, the SDN motif in green.

minimize the energy to regularize local bond and angle geometry, and to relax close contacts in the geometric chain. Each model of VEB-1 was optimized using the variable target function method (VTFM) with conjugate gradients (CG), followed by further refinement using molecular dynamics (MD) with a simulated annealing (SA) method in Modeller [25]. Energy minimization was performed to minimize stearic collisions and strains without significantly altering the overall structure. Energy computations and minimization were carried out using the GROMOS96 force field [26] and implementing Swiss-PdbViewer. After optimization the 3D model of VEB-1 was verified using the PROCHECK [27], ERRAT [28] and VERIFY 3D [29] programs available from the Structural Analysis and Verification Server (SAVES) (http://nihserver.mbi.ucla.edu/SAVES). PROCHECK was used to assess the stereochemical quality of the protein structure, while the Verify3D program analyzed the compatibility of an atomic model (3D) with its own amino acid sequence (1D) to assess the 3D protein structure.

\section{Screening of compounds from the ZINC Database}

Ligand-based virtual screening experiments are important during the early stages of drug discovery, as they can screen compound databases using the active sites of proteins with known 3D structure. The ZINC Database [30] is free to use and contains commercially 
available chemical compounds prepared for virtual screening. It contains more than 21 million compounds in ready-to-dock, 3D formats that can be purchased. During this work the ZINC Database was screened for structurally similar inhibitors of VEB- $1 \beta$-lactamases. The compounds identified included clavulanic acid, sulbactam, tazobactam, imipenem, cefoxitin and moxalactam. Furthermore, this study identified 950 compounds that were structurally similar to available Amber class A $\beta$-lactamase inhibitors during screening.

\section{Structure-based virtual screening using molecular docking}

Virtual screening uses computational methods to identify molecules that are biologically active against a specific protein target [31]. Two types of methodologies can be used during virtual screening: those that search for similarity to validated ligands, and molecular docking methods that require structural information about the target. During this study the first method, which is also known as ligand-based virtual screening, was utilized. Analogs with a minimum of $70 \%$ similarity to the known $\beta$-lactamase inhibitors (clavulanic acid, sulbactam, tazobactam, imipenem, cefoxitin and moxalactam) were selected from the ZINC database. To remove structural redundancies from the chemical library, structurally similar compounds with a Tanimoto coefficient larger than 0.8 were clustered into a single representative molecule. As a consequence, a docking library consisting of 950 compounds was obtained and downloaded in mol2 format.

Virtual screening was performed by docking the inhibitors obtained from the ZINC database to the active site of the VEB-1 protein using AutoDock Vina software (version 1.0) [32]. This docking allowed a population of possible conformations and orientations for the ligand at the binding site to be obtained. Using the Autodock Tools software [33], polar hydrogen atoms were added to VEB-1 protein, and its non-polar hydrogen atoms were merged. The protein receptor (VEB-1) and inhibitors were converted from PDB format to PDBQT format. All bonds within ligands were set to allow rotation. In the configuration file of the Autodock Vina software, a grid box with dimensions of $20 \times 20 \times 20$ points was used around the active site to cover the entire enzyme binding site and allow ligands to move freely.

The docking simulation of each compound was conducted using an improved empirical AutoDock scoring function, in which a new solvation model for organic molecules was introduced. This modified scoring function can be expressed as follows:

$$
\begin{aligned}
& \Delta G_{\text {bind }}^{a q}=W_{v d W} \sum_{i=1} \sum_{j>i}\left(\frac{A_{i j}}{r_{i j}^{12}}-\frac{B_{i j}}{r_{i j}^{6}}\right)+W_{\text {hbond }} \sum_{i=1} \sum_{j>i} E(t)\left(\frac{A_{i j}}{r_{i j}^{12}}-\frac{B_{i j}}{r_{i j}^{6}}\right) \\
& +W_{\text {elec }} \sum_{i=1} \sum_{j>i} \frac{q_{i} q_{i}}{\varepsilon(r i j) r i j}+W_{\text {tor }} N_{\text {tor }}+W_{\text {sol }} \sum_{i=1} S_{i}\left(O c c_{i}^{\max }-\sum_{i>i} V_{j} e^{-\frac{r_{i j}^{2}}{2 \sigma^{2}}}\right)
\end{aligned}
$$

where WvdW, Whbond, Welec, Wtor and Wsol are the weighting factors of van der Waals, hydrogen bond, electrostatic interactions, torsional term and desolvation energy of the inhibitors, respectively. rij represents the interatomic distance, and $A i j, B i j, C i j$ and $D i j$ are related to the depths of the potential energy well and the equilibrium separations between the two atoms. The hydrogen bond term has an additional weighting factor, $E(t)$, representing the angle-dependent directionality. The cubic equation approach was applied to obtain the dielectric constant required for computing the interatomic electrostatic 
interactions between VEB-1 and a ligand molecule [34]. In the entropic term, Ntor is the number of $\mathrm{sp}^{3}$ bonds in the ligand. In the desolvation term, $S i$ and $V i$ are the solvation parameter and the fragmental volume of atom $i$, [35] respectively, while Occi ${ }^{\max }$ is the maximum atomic occupancy. In the calculation of the molecular solvation free energy term in Eq. (1), the atomic parameters developed by Kang et al. [36] were used, as only carbon atoms were available. This modification of the solvation free energy term is expected to increase the accuracy of virtual screening, as underestimation of ligand solvation can lead to overestimation of the binding affinity of a ligand with several polar atoms [37].

The best conformation with the lowest docked energy was chosen from the docking search. The interactions of complex VEB-1 protein-ligand conformations including hydrogen bonds and bond lengths were analyzed using Swiss-PdbViewer v4.0 [38], Pymol software [39], UCSF Chimera [40] and Accelrys DS Visualizer software [41]. The commercially available software toxtree (developed by Idea consult Ltd., Sofia, Bulgaria) was used for computer-based estimation of chemical toxicity [42].

\section{Results and Discussion}

\section{Protein homology modeling and validation}

Multiple sequence alignment of VEB-1 with PER-1, CTX-M-16 and Toho-1 $\beta$-lactamases demonstrated that VEB-1 is highly homologous to PER-1 type $\beta$-lactamases (38\% sequence identity) (Figure 1A). The BLASTP homology search using the E. coli VEB-1 $\beta$ lactamase sequence against the PDB database confirmed this result (data not shown). In addition, sequence alignment indicated that VEB-1 contains a serine-valine-methioninelysine tetrad (SXXK) at positions 70-73, including the conserved serine and lysine amino acid residues that are characteristic of $\beta$-lactamases with a serine active site [43]. Several other structural elements characteristic of class A $\beta$-lactamases were identified including a serine-aspartate-asparagine (SDN) motif at positions 135-137, and lysine-threonine-arginine (KTG) residues at positions 239-242 (Figure 1A.). Accordingly, the crystal structure of PER-1 $\beta$-lactamase from $P$. aeruginosa (PDB ID: 1E25) was used as the template during homology modeling.

The modeled enzyme is a monomer, folded into an $\alpha / \beta$ domain consisting of a sevenstranded $\beta$-sheet and $11 \alpha$-helices (Figure 1B.). The residues in the Ser135-Asp136 -Asn137 (SDN) motif are involved in maintaining the structure of the active site cavity, enzyme stability and stabilization of the enzyme transition state, respectively [44]. Ser135, a conserved amino acid among all class A $\beta$-lactamases, is occasionally replaced by a Gly residue. The multiple roles of this residue include anchoring $\beta$-lactams to the active site, and stabilizing it, through hydrogen bonding with the $\mathrm{C}-3 / \mathrm{C}-4$ carboxylates of inhibitors and substrates and facilitating proton transfer to the $\beta$-lactam nitrogen during acylation, leading to opening of the $\beta$-lactam ring [45].

The quality of the 3D model was evaluated via the Ramachandran plot using PROCHECK software (Figure 2). The Ramachandran plot for the predicted model revealed that $88.2 \%$ of residues were in the most favorable region, while $10.6 \%$ were in the allowed region, confirming that the predicted model is of good quality. ERRAT is a so-called "overall quality factor" for non-bonded atomic interactions, with higher scores indicating higher quality. The generally accepted range is $>50$ for a high quality model. For the current 3D model, the overall quality factor predicted by the ERRAT server was 


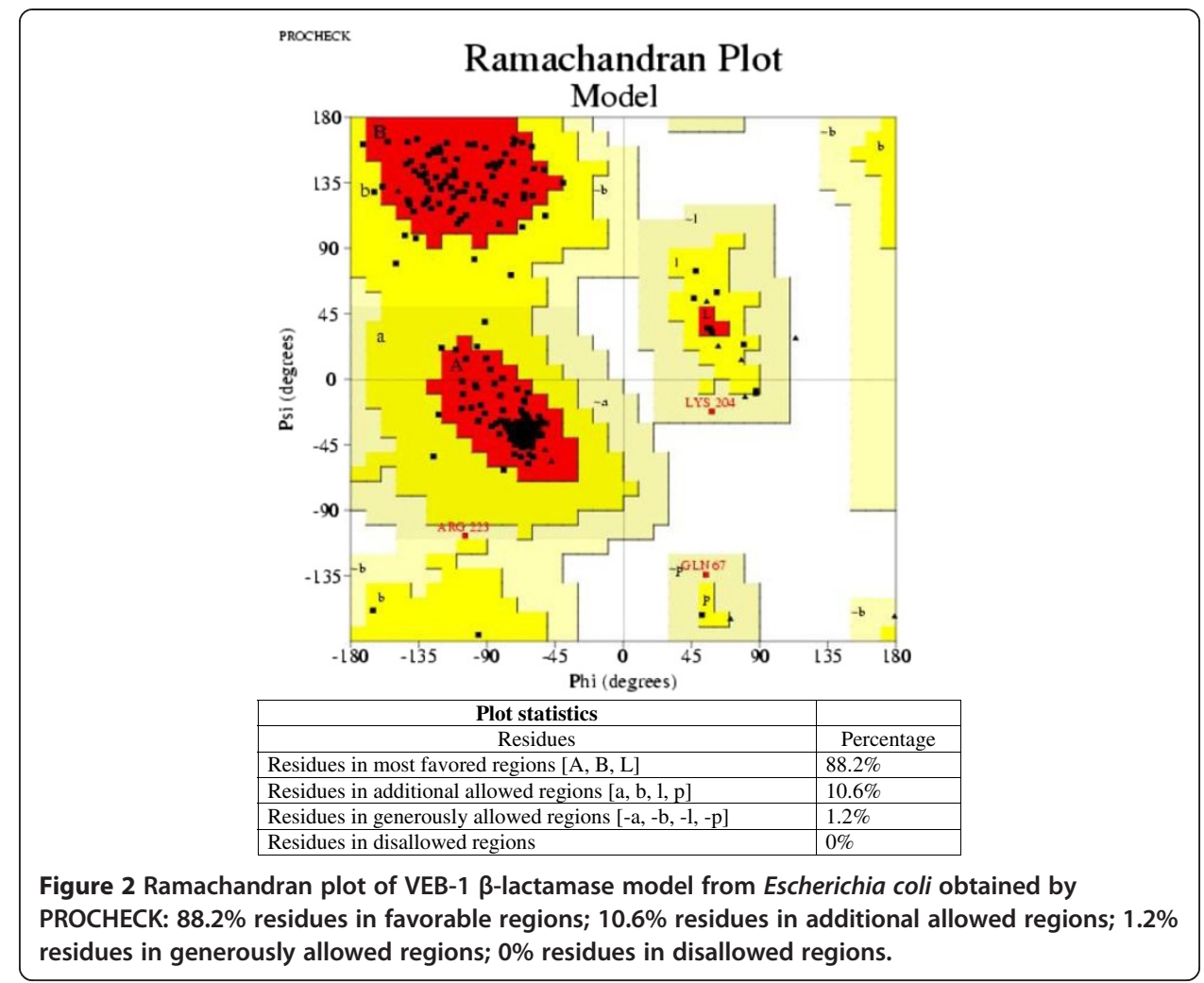

80.524 (Figure 3). The Verify 3D server predicted that $88.77 \%$ of the residues in VEB-1 $\beta$-lactamase had an average $3 \mathrm{D}$-1D score $>0.2$, thereby verifying the model.

\section{Virtual screening result analysis}

Following docking simulations, the four most promising inhibitors were selected on the basis of binding affinity (Figure 4). Among the six categories of Amber class A $\beta$ lactamase inhibitors considered during the analysis, the optimal interactions with the highest affinity scores were obtained with sulbactam analogs. This finding was contrary to previous results for SHV-1 Amber class A $\beta$-lactamase, and sulbactam is a less potent inhibitor than clavulanate [46]. Sulbactam is more potent against class $C \beta$-lactamases than clavulanate, whereas its activity against class $\mathrm{D}$ enzymes is less potent than against class $\mathrm{A}$ $\beta$-lactamases. Similarly, sulbactam does not inhibit OXA-type enzymes as efficiently as TEM-1 and other clinically used inhibitors [47].

The best conformation demonstrated that the free energy of binding ( $\Delta \mathrm{Gbind}, \mathrm{kcal} / \mathrm{mol}$ ) for the top four inhibitors was good. The negative and low value of $\Delta$ Gbind (-6.4) indicated strong bonds between VEB-1 and the ZINC4085364 inhibitor, and demonstrated that the inhibitor was in its most favorable conformation. Analysis of the docked complexes demonstrated that the inhibitor was located close to the active site (Ser68), at a distance of $0.6 \AA$. The complex was stabilized by four hydrogen bonds through residues Ser68, Lys71, Ser131 and Gln67 (Figure 5B). The residue involved in cavity formation is presented in Figure 5A. Interaction analysis revealed that the cavity involved in the 


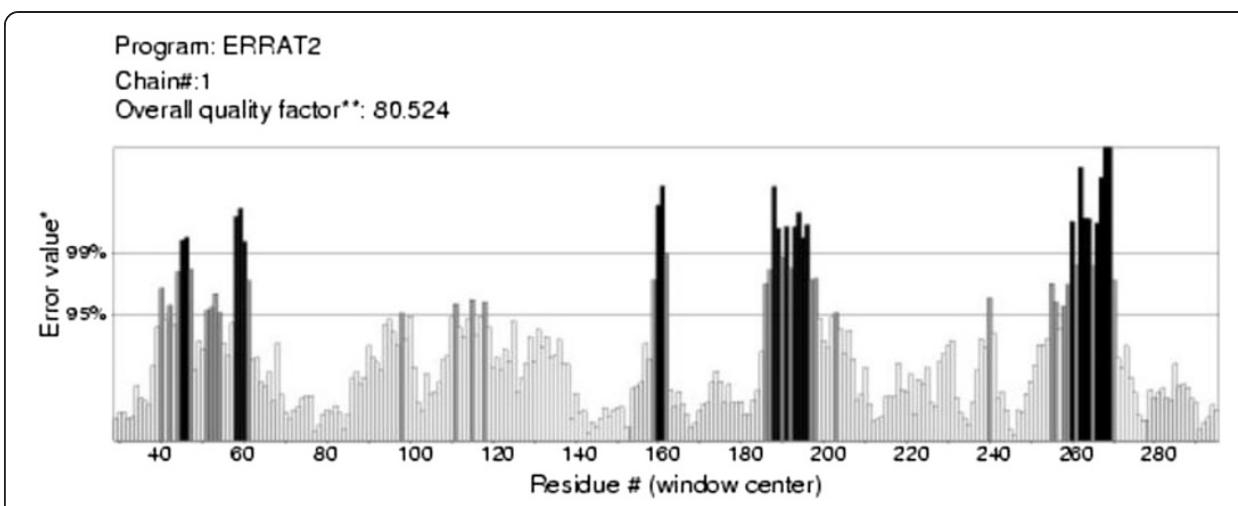

Figure 3 Errat plot for the VEB-1 $\beta$-lactamase model. Black bars identify the misfolded region located distantly from the active site, gray bars demonstrate the error region between $95 \%$ and $99 \%$, and white bars indicate the region with a lower error rate for protein folding.

binding site has a volume of $186.6 \AA^{3}$ and a surface area of $178.2 \AA^{2}$. Toxtree was used to estimate toxic properties. Finally, four molecules were selected (Table 1).

\section{Conclusions}

Antibiotic resistance is one of the most serious threats to public health. Development of resistance is assisted by the existence of plasmids, which can be transmitted easily between bacteria. The emergent VEB-1 $\beta$-lactamase possesses potent hydrolysis activity

\begin{tabular}{|l|l|l|l|}
\hline S.No & Ligands (ZINC ID) & Structure of molecule & \multicolumn{1}{c|}{ Binding Affinity } \\
(Kcal/mol)
\end{tabular}

Figure 4 Estimated Free Energy of Binding of the top four ligands. 

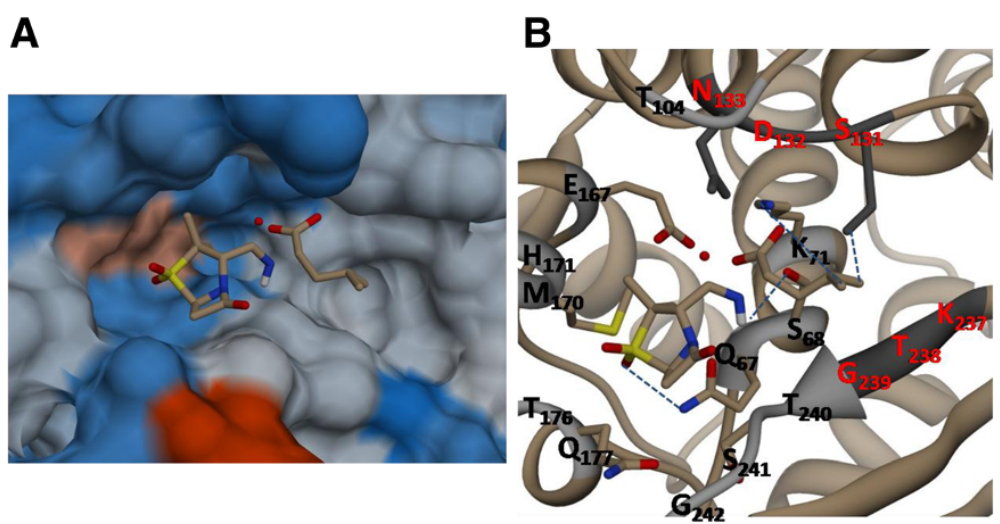

Figure 5 Binding interactions of inhibitors demonstrating maximum binding affinity (ZINC4085364), and the modeled VEB-1 protein. The left presents the structural electrostatic surface, and the right the detailed binding interactions. (hydrogen bond interactions are indicated with blue dotted lines).

towards almost all antibiotics and is a significant threat. Virtual screening is an important tool for exploring biologically relevant chemical spaces, and allows studies focused on small molecule libraries to be performed, using up to millions of compounds. During the present study, structural models of a VEB-1/ZINC4085364 inhibitor complex were obtained using homology modeling and molecular docking methods. At present, there are no effective antibiotics against VEB-1-positive pathogens. An appropriate strategy involves identifying drug candidates from existing antibiotics, such as cephalosporin, on the basis of the 3D model of VEB-1 using structure-based virtual screening. This strategy was used successfully in the discovery of Merck's HIV protease inhibitor [48]. The molecule identified in the current study as a VEB-1 inhibitor could be exploited for drug design. However, further in vivo experimentation is required for complete evaluation.

Table 1 Physiochemical properties of the most potent ligand (ZINC4085364) obtained from the docking study

\begin{tabular}{ll}
\hline pH range & Reference $\mathbf{( p H ~ 7 )}$ \\
\hline xlogP & -1.64 \\
\hline Apolar desolvation $(\mathrm{kcal} / \mathrm{mol})$ & -7.31 \\
\hline Polar desolvation $(\mathrm{kcal} / \mathrm{mol})$ & -66.16 \\
\hline H-bond donors & 1 \\
\hline H-bond acceptors & 8 \\
\hline Net charge & -1 \\
\hline tPSA $\left(\AA^{2}\right)$ & 123 \\
\hline Molecular weight $(\mathrm{g} / \mathrm{mol})$ & 345.397 \\
\hline Rotatable bonds & 5 \\
\hline Popular name & (S)-2-((2S,5R)-3,3-dimethyl-4,4-dioxido-7-oxo-4-thia-1-azabicyclo[3.2.0] \\
\hline Molecular formula & heptane-2-carboxamido)-4-methylpentanoic acid \\
\hline
\end{tabular}


The authors declare that they have no competing interests.

\section{Authors' contributions}

AM carried out all analyses and drafted the manuscript under the guidance of $\mathrm{HB}$ and JBH. All authors read and approved the final manuscript.

Received: 13 February 2013 Accepted: 23 March 2013

Published: 2 April 2013

\section{References}

1. Poirel L, Naas T, Guibert M, Chaibi EB, Labia R, Nordmann P: Molecular and biochemical characterization of VEB-1, a novel class A extended-spectrum $\beta$-lactamase encoded by an Escherichia coli integron gene. Antimicrob Agents Chemother 1999, 43:573-581.

2. Naas T, Mikami Y, Imai T, Poirel L, Nordmann P: Characterization of In53, a class 1 plasmid and composite transposon-located integron of Escherichia coli which carries an unusual array of gene cassettes. J Bacteriol 2001, 183:235-249.

3. Naas T, Poirel L, Nordmann P: Minor extended-spectrum $\beta$-lactamases. Clin Microbiol Infect 2008, 14(Suppl.1):42-52.

4. Poirel L, Villa L, Bertini A, Pitout JD, Nordmann P, Carattoli A: Expanded-spectrum $\beta$-lactamase and plasmidmediated quinolone resistance. Emerg Infect Dis 2007, 13:803-805.

5. Adams MD, Goglin K, Molyneaux N, Hujer KM, Lavender H, Jamison JJ, MacDonald IJ, Martin KM, Russo T, Campagnari AA, Hujer AM, Bonomo RA, Gill SR: Comparative genome sequence analysis of multidrug-resistant Acinetobacter baumannii. J Bacteriol 2008, 190:8053-8064.

6. Aragon LM, Mirelis B, Miro E, Mata C, Gomez L, Rivera A, Coll P, Navarro F: Increase in $\beta$-lactam-resistant Proteus mirabilis strains due to CTX-M- and CMY-type as well as new VEB-1 and inhibitor-resistant TEM-type $\beta$ lactamases. J Antimicrob Chemother 2008, 61:1029-1032.

7. Girlich D, Poirel L, Leelaporn A, Karim A, Tribuddharat C, Fennewald M, Nordmann P: Molecular epidemiology of the integronlocated VEB-1 extended-spectrum $\beta$-lactamase in nosocomial enterobacterial isolates in Bangkok, Thailand. J Clin Microbiol 2001, 39:175-182.

8. Poirel L, Pitout JD, Calvo L, Rodriguez-Martinez JM, Church D, Nordmann P: In vivo selection of fluoroquinoloneresistant Escherichia coli isolates expressing plasmid-mediated quinolone resistance and expanded-spectrum ß-lactamase. Antimicrob Agents Chemother 2006, 50:1525-1527.

9. Toleman MA, Bennett PM, Walsh TR: Common regions e.g. orf513 and antibiotic resistance: IS91-like elements evolving complex class 1 integrons. J Antimicrob Chemother 2006, 58:1-6.

10. Lahlaoui H, Poirel L, Moussa MB, Ferjani M, Omrane B, Nordmann P: Nosocomial dissemination of extendedspectrum $\beta$-lactamase VEB-1a-producing Providencia stuartii isolates in a Tunisian Hospital. Eur $J$ Clin Microbio Infect Dis 2011, 30:1267-1270.

11. Aubert $D$, Girlich $D, N$ aas $T$, Nagarajan $S$, Nordmann P: Functional and structural characterization of the genetic environment of an extended-spectrum $\beta$-lactamase blaVEB gene from a Pseudomonas aeruginosa isolate obtained in India. Antimicrob Agents Chemother 2004, 48:3284-3290.

12. Poirel L, Rotimi VO, Mokaddas EM, Karim A, Nordmann P: VEB-1-like extended-spectrum $\beta$-lactamases in Pseudomonas aeruginosa. Kuwait Emerg Infect Dis 2001, 7:468-470.

13. Nordmann P, Naas T: Sequence analysis of PER-1 extended-spectrum beta-lactamase from Pseudomonas aeruginosa and comparison with class A b-lactamases. Antimicrob Agents Chemother 1994, 38:104-114.

14. Naas T, Aubert D, Lambert T, Nordmann P: Complex genetic structures with repeated elements, a sul-type class 1 integron, and the blaVEB extended-spectrum b-lactamase gene. Antimicrob Agents Chemother 2006, 50:1745-1752.

15. Reading C, Cole M: Clavulanic acid: a $\beta$-lactamase-inhibiting $\beta$-lactam from Streptomyces clavuligerus. Antimicrob Agents Chemother 1977, 11:852-857.

16. Brown AG: Clavulanic acid, a novel $\beta$-lactamase inhibitor a case study in drug discovery and development. Drug Des Deliv 1986, 1:1-21

17. English AR, Retsema JA, Girard AE, Lynch JE, Barth WE: CP-45,899, a $\beta$-lactamase inhibitor that extends the antibacterial spectrum of $\beta$-lactams: initial bacteriological characterization. Antimicrob Agents Chemother 1978, 14:414-419.

18. Fisher JJ, Belasco G, Charnas RL, Khosla S, Knowles JR: $\beta$-Lactamase inactivation by mechanism-based reagents. Philos Trans R Soc Lond 1980, 289:309-319.

19. Berman HM, Westbrook J, Feng Z, Gilliland G, Bhat TN, Weissig H, Shindyalov IN, Bourne PE: The protein data bank. Nucleic Acids Res 2000, 28:235-242.

20. Altschul SF, Madden TL, Schäffer AA, Zhang J, Zhang Z, Miller W, Lipman DJ: Gapped BLAST and PSI-BLAST: a new generation of protein database search programs. Nucleic Acids Res 1997, 25:3389-3402.

21. Tranier S, Bouthors AT, Maveyraud L, Guillet V, Sougakoff W, Samama JP: The high resolution crystal structure for class A beta-lactamase PER-1 reveals the bases for its increase in breadth of activity. J Biol Chem 2000, 275:28075-28082.

22. Schwede T, Kopp J, Guex N, Peitsch MC: SWISS-MODEL: an utomated protein homology-modeling server. Nucleic Acids Res 2003, 31:3381-3385.

23. Gasteiger E, Gattiker A, Hoogland C, Ivanyi I, Appel RD, Bairoch A: ExPASy: the proteomics server for in-depth protein knowledge and analysis. Nucleic Acids Res 2003, 31:3784-3788.

24. Arnold K, Bordoli L, Kopp J, Schwede T: The SWISS-MODEL Workspace: A web-based environment for protein structure homology modeling. Bioinformatics 2006, 22:195-201. 
25. Šali A, Blundell TL: Comparative protein modelling by satisfaction of spatial restraints. J Mol Biol 1993, 234:779-815.

26. Scott W, Huenenberger PH, Tironi IG, Mark AE, Billeter SR, Fennen J, Torda AE, Huber T, Krueger P, van Gunsteren WF: The GROMOS Biomolecular Simulation Program Package. J Phys Chem A 1999, 103:3596-3607.

27. Laskowski RA, MacArthur MW, Moss DS, Thornton JM: PROCHECK a program to check the stereochemical quality of protein structures. J App/ Crystallogr 1993, 26:283-291.

28. Colovos C, Yeates TO: Verification of protein structures: patterns of nonbonded atomic interactions. Protein SCi 1993, 2:1511-1519

29. Bowie JU, Lüthy R, Eisenberg D: A method to identify protein sequences that fold into a known threedimensional structure. Science 1991, 253:164-170.

30. Irwin JJ, Shoichet BK: ZINC: A free database of commercially available compounds for virtual screening. $J$ Chem Inf Model 2005, 45:177-182.

31. Vianna CP, Azevedo WF: Identification of new potential Mycobacterium tuberculosis shikimate kinase inhibitors through molecular docking simulations. J Mol Model 2012, 18:755-764.

32. Trott O, Olson AJ: AutoDock Vina: improving the speed and accuracy of docking with a new scoring function efficient optimization and multithreading. J Comput Chem 2010, 31:455-461.

33. Goodsell DS, Olson AJ: Automated docking of substrates to proteins by simulated annealing. Proteins 1990 8:195-202.

34. Park H, Jeon JH: Cubic equation governing the outer-region dielectric constant of globular proteins. Phys Rev E 2007, 75:021916.

35. Stouten PFW, Frömmel C, Nakamura H, Sander C: An Effective Solvation Term Based on Atomic Occupancies for Use in Protein Simulations. Mol Simul 1993, 10:97-120.

36. Kang H, Choi H, Park H: Prediction of Molecular Solvation Free Energy Based on the Optimization of Atomic Solvation Parameters with Genetic Algorithm. J Chem Inf Model 2007, 47:509-514.

37. Shoichet BK, Leach AR, Kuntz ID: Ligand solvation in molecular docking. Proteins 1999, 34:4-16.

38. Guex N, Peitsch MC: SWISS-MODEL and the Swiss-PdbViewer: An environment for comparative protein modeling. Electrophoresis 1997, 18:2714-2723.

39. DeLano WL: The PyMOL molecular graphics system DeLano Scientific LLC. CA, USA: San Carlos; 2002.

40. Pettersen EF, Goddard TD, Huang CC, Couch GS, Greenblatt DM, Mengb EC, Ferrin TE: UCSF Chimera - a visualization system for exploratory research and analysis. J Comput Chem 2004, 25:1605-1612.

41. The Accelrys DS Visualizer software. http://accelrys.com.

42. Wolber G, Seidel T, Bendix F, Langer T: Molecule-pharmacophore superpositioning and pattern matching in computational drug design. Drug Discov Today 2008, 13:23-29.

43. Joris B, Ledent P, Dideberg O, Fonze E, Lamotte-Brasseur J, Kelly JA, Ghuysen JM, Frere JM: Comparison of the sequences of classA b-lactamases and of the secondary structure elements of penicillin-recognizing proteins. Antimicrob Agents Chemother 1991, 35:2294-2301.

44. Jacobs $C$, Frere JM, Normark S: Cytosolic intermediates for cell wall biosynthesis and degradation control inducible $\beta$-lactam resistance in gram-negative bacteria. Cell 1997, 88:823-832.

45. Imtiaz U, Billings EM, Knox JR, Manavathu EK, Lerner SA, Mobashery S: Inactivation of class A $\beta$-lactamases by clavulanic acid: the role of Arginine 244 in a proposed non certed sequence of events. J Am Chem Soc 1993, 115:4435-4442.

46. Bush K, Macalintal C, Rasmussen BA, Lee VJ, Yang Y: Kinetic interactions of tazobactam with b-lactamases from all major structural classes. Antimicrob Agents Chemother 1993, 7:851-858.

47. Akova M: Sulbactam-containing b-lactamase inhibitor combinations. Clin Microbiol Infect 2008, 14:185-188

48. Des Jarlais RL, Seibel GL, Kuntz ID, Furth PS, Alvarez JC, Ortiz De Montellano PR, De Camp DL, Babé LM, Craik CS: Structure-based design of non peptide inhibitors specific for the human immunodeficiency virus 1 protease. Proc Natl Acad Sci USA 1990, 87:6644-6648.

doi:10.1186/1742-4682-10-22

Cite this article as: Messaoudi et al:: Homology modeling and virtual screening approaches to identify potent inhibitors of VEB-1 $\beta$-lactamase. Theoretical Biology and Medical Modelling 2013 10:22.

\section{Submit your next manuscript to BioMed Central and take full advantage of:}

- Convenient online submission

- Thorough peer review

- No space constraints or color figure charges

- Immediate publication on acceptance

- Inclusion in PubMed, CAS, Scopus and Google Scholar

- Research which is freely available for redistribution 